\title{
Perspective
}

Actualité en histoire de l'art

\section{Mode et propagande. Les décors privés et la révolution culturelle augustéenne}

Fashion and Propaganda. Domestic Interiors and the Augustan Cultural

Revolution

Mode und Propaganda. Private Ausstattung und die augusteische

Kulturrevolution

Moda e propaganda. Le decorazioni private e la rivoluzione culturale augustea

Moda y propaganda. El diseño de interiores y la revolución cultural agustina

\section{Anna Anguissola}

Traducteur : Françoise Jaouën

\section{OpenEdition}

\section{Journals}

Édition électronique

URL : https://journals.openedition.org/perspective/7270

DOI : $10.4000 /$ perspective. 7270

ISSN : 2269-7721

Éditeur

Institut national d'histoire de l'art

\section{Édition imprimée}

Date de publication : 30 juin 2017

Pagination : 145-152

ISBN : 9782917902387

ISSN : $1777-7852$

\section{Référence électronique}

Anna Anguissola, " Mode et propagande. Les décors privés et la révolution culturelle augustéenne », Perspective [En ligne], 1 | 2017, mis en ligne le 31 décembre 2017, consulté le 16 janvier 2023. URL: http://journals.openedition.org/perspective/7270 ; DOI : https://doi.org/10.4000/perspective.7270 


\section{Mode et propagande. Les décors privés et la révolution culturelle augustéenne}

\section{Anna Anguissola}

- ClARKe, 2007 : John R. Clarke, «Augustan Domestic Interiors: Propaganda or Fashion? " dans Karl Galinsky (dir.), The Cambridge Companion to the Age of Augustus, Cambridge/New York, Cambridge University Press, 2007, p. 264-280.

- Milnor, 2005 : Kristina Milnor, Gender, Domesticity, and the Age of Augustus: Inventing Private Life, Oxford/ New York, Oxford University Press, 2005. XII + 360 p.

- WALlACe-HAdRILl, 2008 : Andrew Wallace-Hadrill, Rome's Cultural Revolution, Cambridge/New York, Cambridge University Press, 2008. XXI + 502 p.

Trente ans se sont écoulés depuis que Paul Zanker a montré comment les images ont stimulé, favorisé et diffusé la transition entre l'atmosphère de pessimisme qui régnait dans l'État romain lorsque Auguste a commencé à asseoir son pouvoir et "l'optimisme et la confiance qui, en trouvant leur expression sous la plume des poètes augustéens, léguèrent à la postérité le concept du Saeculum Augustum ${ }^{1}$ ".

Zanker s'intéressait avant tout, non pas aux monuments et aux traditions artistiques, mais aux contextes sociaux et politiques dans lesquels émerge un " langage visuel ». La notion de renovatio - la volonté de remodeler la culture du présent selon un passé idéalisé - est au fondement du « langage visuel » augustéen, dont les évolutions au niveau du contenu et du style incarnent un choix politique et moral ${ }^{2}$. Ce n'est pas un hasard si l'année même où est publié le texte fondateur de Zanker, Augustus und die Macht der Bilder (1987), Tonio Hölscher fait paraître un autre ouvrage capital intitulé Römische Bildsprache als semantisches System, dont l'argument repose sur la même prémisse, à savoir que les choix stylistiques renferment des valeurs morales ${ }^{3}$. $\mathrm{Au}$ sein de ce système sémantique, le style archaïque représente la pietas, tandis que celui du V viècle incarne l'auctoritas et la maiestas. En privilégiant les formes archaïques et classiques, on choisit ainsi un ensemble précis de valeurs correspondant à un programme idéologique centré sur certaines qualités. Le programme augustéen de renouveau culturel, mis au point dans le cercle proche de l'empereur, s'étend rapidement à travers l'Empire et imprègne toute la société romaine. Le paysage urbain de Rome est profondément altéré afin de marquer ses édifices et espaces publics du sceau idéologique du prince. Parallèlement, une imagerie nouvelle s'impose, qui pénètre tous les aspects de la vie publique et privée. Rome subit une métamorphose spectaculaire ${ }^{4}$, troquant ses bâtiments traditionnels en brique contre du marbre poli. Les nouveaux monuments consolident les revendications dynastiques et la vision politique d'Auguste. Hors de Rome, les élites locales s'empressent d'adopter et de diffuser l'ensemble de références et de valeurs dominantes, et prennent une part essentielle au remaniement de leurs villes et de leurs sanctuaires ${ }^{5}$. La nouvelle idéologie se reflète également dans la sphère privée, tant dans la conception de l'habitat que dans la dynamique de la vie quotidienne.

L'art privé adopte et banalise les caractéristiques et les symboles des monuments impériaux, ce qui démontre l'ampleur de la diffusion du « langage visuel " augustéen. Comme on l'a souvent souligné, c'est sur ce point que le livre de Zanker présente l'une de ses plus grandes originalités. La notion traditionnelle de "propagande " (imposée par le pouvoir en place) s'efface au profit d'un tableau plus nuancé qui tient compte de la capacité des sujets et des artistes à répondre aux innovations sociales, culturelles et artistiques introduites par l'empereur. L'imagerie et la production artistique du règne d'Auguste ne sont pas les produits d'une "machine de propagande en action ", mais constituent une réponse fluide à des facteurs multiples et 
1. Détail du décor peint de la « chambre noire ", villa de Boscotrecase, troisième style, New York, The Metropolitan Museum of Art.

parfois contradictoires : communications officielles à divers niveaux, souci de l'image donnée par la famille, attentes collectives, ambitions individuelles et autoreprésentation ${ }^{6}$.

Dans ce cadre, le ton donné par le prince et son entourage peut faire l'objet d'ajustements spontanés, ou au contraire délibérés et réfléchis. À leur tour, les images ou les œuvres littéraires qui se conforment au nouvel ensemble de valeurs deviennent essentielles à la dissémination de celles-ci.

Le décor de troisième style s'inscrit dans le nouveau canon esthétique et moral, simultanément foisonnant et soigneusement ordonné ${ }^{7}$. Les exubérants schémas illusionnistes reposant sur les perspectives architecturales, les entablements, les pilastres, les colonnes et les moulures, répandus quelques années plus tôt, laissent place à de délicats motifs ornementaux presque immatériels. Si l'on peut considérer que ces éléments d'encadrement représentent plutôt une mise à jour de la tradition des paysages architecturaux peints, les vastes surfaces monochromes constituent en revanche une véritable innovation. La récurrence des candélabres, des paysages sacrés et des scènes pastorales bucoliques aux accents religieux correspondent parfaitement à l'esprit et au goût de la période.

Dans une petite ville telle que Pompéi, même les maisons modestes participent à ce changement radical en remettant au goût du jour leurs peintures murales. Les élites, quant à elles, adoptent sans réserve le nouveau style, appliqué à l'ensemble du système décoratif de leurs magnifiques villas. Le décor raffiné de la villa de Boscotrecase, qui pourrait avoir appartenu à la famille d'Agrippa, est un chef-d'œuvre de peinture romaine du troisième style ${ }^{8}$ (fig. 1). Chacun des éléments du décor de la " chambre noire " répond à un désir de clarté et d'uniformité. Au-dessus d'un soubassement rouge, de frêles colonnes blanches tracées sur un fond noir lustré soutiennent des silhouettes de pavillons, de candélabres et de trépieds. De minuscules motifs enjolivent leurs troncs aériens. De délicats cygnes, symboles d'Apollon, le dieu tutélaire d'Auguste, retiennent des festons diaphanes. Dans le registre supérieur du mur on trouve des panneaux montrant des scènes égyptisantes sur fond jaune, qui font peut-être allusion à la récente annexion de l'Égypte mais qui renvoient, de manière plus générale à l'horizon élargi de la culture de l'époque. De minuscules vignettes de paysages où se déroulent des rites sacrés, se détachent du fond noir sans aucun relief.

Trois décennies après la parution du livre de Zanker, la complexité du programme augustéen, qui s'applique à l'ensemble de la culture matérielle sous tous ses aspects, reste un élément clef pour comprendre, non seulement la production littéraire et artistique de l'époque, mais aussi, et surtout, les multiples liens entre une société et son passé. Les questions soulevées par la rigoureuse argumentation de Zanker n'ont cessé d'occuper les spécialistes de la Rome augustéenne, comme en témoignent les nombreux ouvrages - biographies, manuels et catalogues d'exposition - publiés à l'occasion du bimillénaire de la mort d'Auguste (2014), et qui se sont tous confrontés à l'épineuse question de la politique et de la propagande culturelles. Concernant l'histoire sociale et culturelle de la période, ce sont avant tout les grands travaux publics et le remaniement architectural de Rome qui ont récemment suscité le plus d'intérêt. Toutefois, les mécanismes d'assimilation du "langage visuel " augustéen restent tout aussi pertinents s'agissant de l'étude de décors intérieurs, notamment dans les villes de Campanie et des alentours détruites par l'éruption du Vésuve en 79 av. notre ère.

Ces contextes géographiques fournissent de précieuses informations sur la réception des nouveaux goûts et modèles, à la fois dans les luxueuses villas des élites et dans les maisons plus modestes de la classe moyenne urbaine. La conception des décors peints, mais aussi le type d'objets du quotidien, leur forme, les matériaux utilisés, leur provenance et leur style témoignent d'un profond bouleversement dans la construction de l'identité 
et la réponse apportée au système de savoir dominant. Il est bien entendu impossible d'évoquer ici les multiples significations du " goût " ni la dynamique sociale que le concept recouvre en termes de subjectivité, de normes et de valeurs. Mais nombre d'études récentes consacrées à la culture matérielle de la période augustéenne se penchent, de manière plus ou moins implicite, sur la définition du goût et son impact sur la culture visuelle dans la société romaine, études qui sont au cœur de cette brève contribution.

\section{Les " vagues de mode "}

Les intérieurs privés constituent un sujet d'étude privilégié pour comprendre la société de la période, en raison d'une tension présente dans le programme augustéen, et jusqu'ici irrésolue. L'étalage du pouvoir et la consommation ostentatoire migrent vers la sphère privée lorsque les ambitieux projets urbains deviennent l'apanage du prince, dépouillant ainsi les élites des moyens d'autoreprésentation qui caractérisaient la fin de la République. La possession et l'exhibition d'objets de luxe deviennent une forme d'expression de la condition sociale et de l'identité, phénomène alimenté par les besoins des élites " emprisonnées dans le goulot du système politique romain ", désigné chez Andrew Wallace-Hadrill par l'expression de "révolution de la consommation" " (WALLACE-HADRILL, 2008, p. 450). Les nouveaux produits de luxe, comme l'a montré Zanker, ne restent pas longtemps le privilège des élites, et se diffusent dans l'ensemble de la société, souvent sous des formes simplifiées et banalisées (fig. 2). Selon Wallace-Hadrill, la " mode " advient par " vagues ", suivant des rythmes réguliers d'assimilation et d'appropriation. Le processus décrit par Pline l'Ancien, à savoir que différents types de marchandises de luxe sont arrivées à Rome par vagues successives dans le sillage de la conquête, est développé en trois étapes : introduction, appropriation et émulation ${ }^{10}$. Au cours de ce processus, le marché des produits destinés aux classes aisées se transforme en une production de masse d'artefacts copiant plus ou moins les objets très enviés possédés par les élites, et qui deviennent ainsi des "imitations de luxe bas de gamme ". D'une certaine manière, la rapide expansion de Rome et l'introduction de produits étrangers qui s'ensuit satisfait la demande de nouveauté intrinsèque à la mode. Une fois assimilé, le produit ou l'habitude évolue de manière autonome,

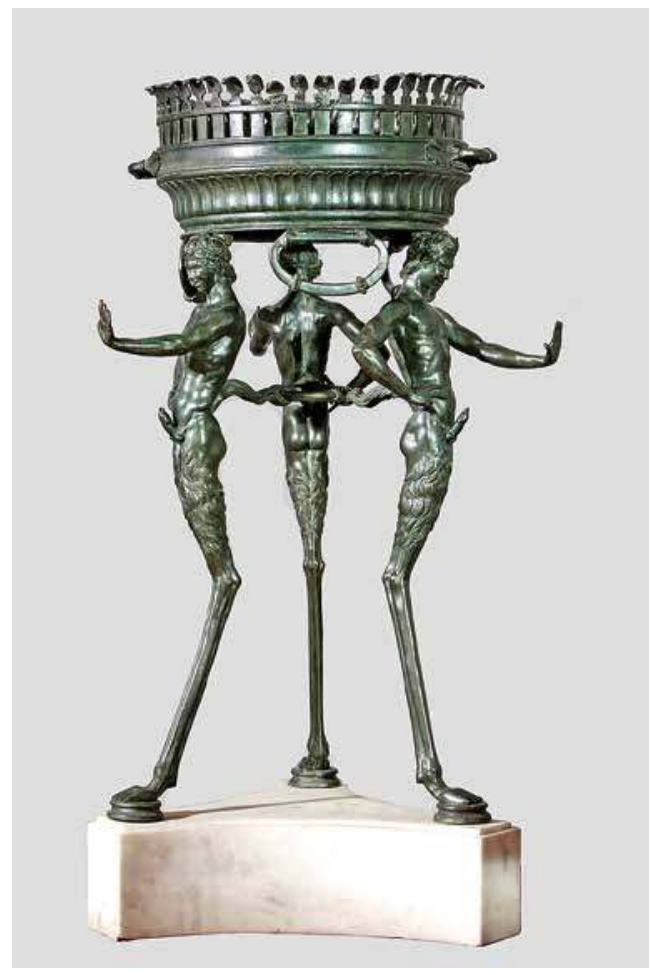

2. Trépied avec satyres ithyphalliques, Pompéi, période augustéenne,

Naples, Museo Archeologico Nazionale.

indépendamment de toute motivation subjective, et devient un instrument banalisé de communication sociale et institutionnelle ${ }^{11}$.

La peinture de jardins et l'introduction de motifs égyptisants dans la décoration murale de troisième style sont deux exemples de ces « vagues de mode " qui semblent avoir pris naissance dans les milieux de la cour, mais se sont rapidement propagées à l'ensemble de la société romaine. La vogue des jardins peints va de pair avec l'évolution de la conception et de l'entretien des jardins et un goût de plus en plus prononcé pour les grottes naturelles et artificielles, à la fois ornements et lieux de retraite ${ }^{12}$.

On a découvert dans la somptueuse maison du Bracelet d'or à Pompéi de magnifiques salles panoramiques ornées de jardins peints ${ }^{13}$. Les classes moyennes de la ville pouvaient elles aussi s'offrir au moins une pièce décorée selon ce nouveau goût. C'est notamment le cas de la maison du Verger qui, loin de la magnificence des demeures des élites locales ou du luxe extravagant des vastes villas telles que celle d'Agrippa Postumus à Boscotrecase, possédait néanmoins une décoration raffinée intégrant les nouveaux modèles ${ }^{14}$. La présence de motifs pharaoniens, parfois mal interprétés, et leur fréquente utilisation 
3. Torse, fragment d'une copie du Doryphore, période augustéenne, Florence, Galleria degli Uffizi.

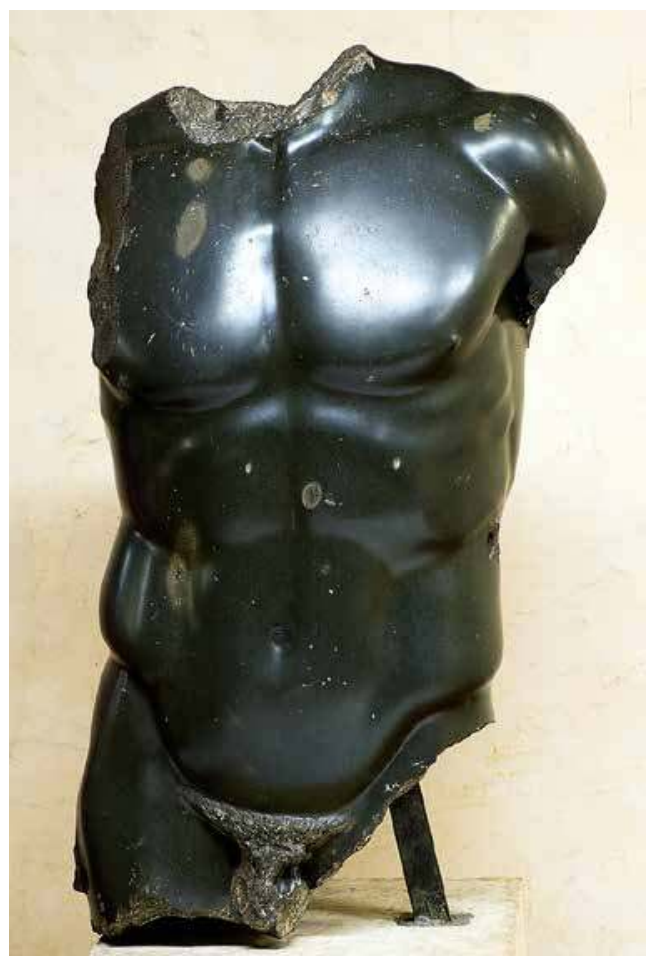

décorative combinés à d'autres thèmes, montrent que l'imagerie égyptienne a été largement assimilée, absorbée dans le nouveau savoir et intégrée dans un nouveau contexte géographique, matériel, social et culturel ${ }^{15}$. Les jardins peints comme ceux du décor de la maison du Bracelet d'or, ou encore ceux de la maison du Verger, démontrent que différentes "vagues de mode " se sont parfois combinées pour donner naissance à des environnements à multiples facettes, mais cohérents. Le goût pour les décors de jardins, la passion pour les symboles égyptiens et la mythologie grecque s'inscrivent dans un " langage visuel " qui a parfaitement assimilé les leitmotive de la propagande augustéenne. C'est également le cas des nombreuses copies de célèbres prototypes sculptés du passé classique ${ }^{16}$. Celle du Doryphore conservé à la Galleria degli Uffizi, exécutée en basalte égyptien poli (inv. $n^{\circ} 308$ ), et dont seul le torse a survécu, évoquait sans doute simultanément l'ensemble de valeurs incarnée par le porte-lance de Polyclète et le contrôle exercé par la Rome d'Auguste sur les ressources naturelles de l'Égypte (fig. 3).

Les élites et les nouveaux groupes sociaux émergents trouvent dans l'exhibition des richesses un langage permettant d'affirmer leur identité à deux niveaux : individus ayant les moyens de s'offrir des objets précieux, ils sont également des acteurs dans le projet romain de conquête. Dans ce cadre, les lois somptuaires, tout en garantissant que la concentration de richesses ne nuise pas à l'ordre social établi, limitent l'accès aux produits de luxe, qui n'en deviennent que plus convoités ${ }^{17}$. D'un côté, le luxe, lieu principal d'une compétition incontrôlée, est un facteur potentiel de trouble social. De l'autre, les mécanismes d'émulation qu'il déclenche constituent la prémisse nécessaire à la diffusion du nouveau " langage visuel " et du programme idéologique qu'il porte. WallaceHadrill privilégie le discours des " rythmes sousjacents ", au détriment de l'action délibérée des individus qui produisent, échangent, achètent et contemplent les diverses catégories d'objets. En tout état de cause, ce processus d'assimilation fonctionne comme un moyen d'expression et de validation aussi bien de l'identité individuelle que collective ; la distinction entre ce qui est implicite et inconscient, et ce qui est reconnu et accepté en connaissance de cause en résulte brouillée.

\section{Mode ou propagande ?}

Dans l'analyse de Wallace-Hadrill, une question fondamentale reste partiellement sans réponse : où se trouve la limite entre la propagande et la mode ? Où se termine la propagande - la transmission délibérée, par le pouvoir en place, de messages à portée culturelle - et où commence la mode - l'adoption quasi-généralisée de marchandises et de comportements en tant que marqueurs de capital social ? Plus précisément, comment les nouveaux systèmes décoratifs s'inscrivent-ils dans le programme général de renouveau culturel et religieux mis en place par Auguste (fig. 4) ? Les commanditaires et les artistes à l'origine des nouveaux systèmes décoratifs des murs et des plafonds de Pompéi " prêchent-ils l'idéologie néoclassique augustéenne marquée par la piété et la mesure, ou suivent-ils simplement une nouvelle vogue élégante, et souvent copiée, de décoration intérieure $^{18}$ " ? John R. Clarke sait pertinemment que, pour répondre à cette question et en dévoiler les présupposés, il faut au préalable distinguer l'art de la mode. Cette distinction entre, d'un côté "l'art noble ", qui s'incarne dans " les œuvres des artistes grecs rapportées et exposées à Rome " et, de l'autre, les "peintures, stucs et mosaïques " exécutés "par de talentueux artisans soucieux de fournir un cadre 


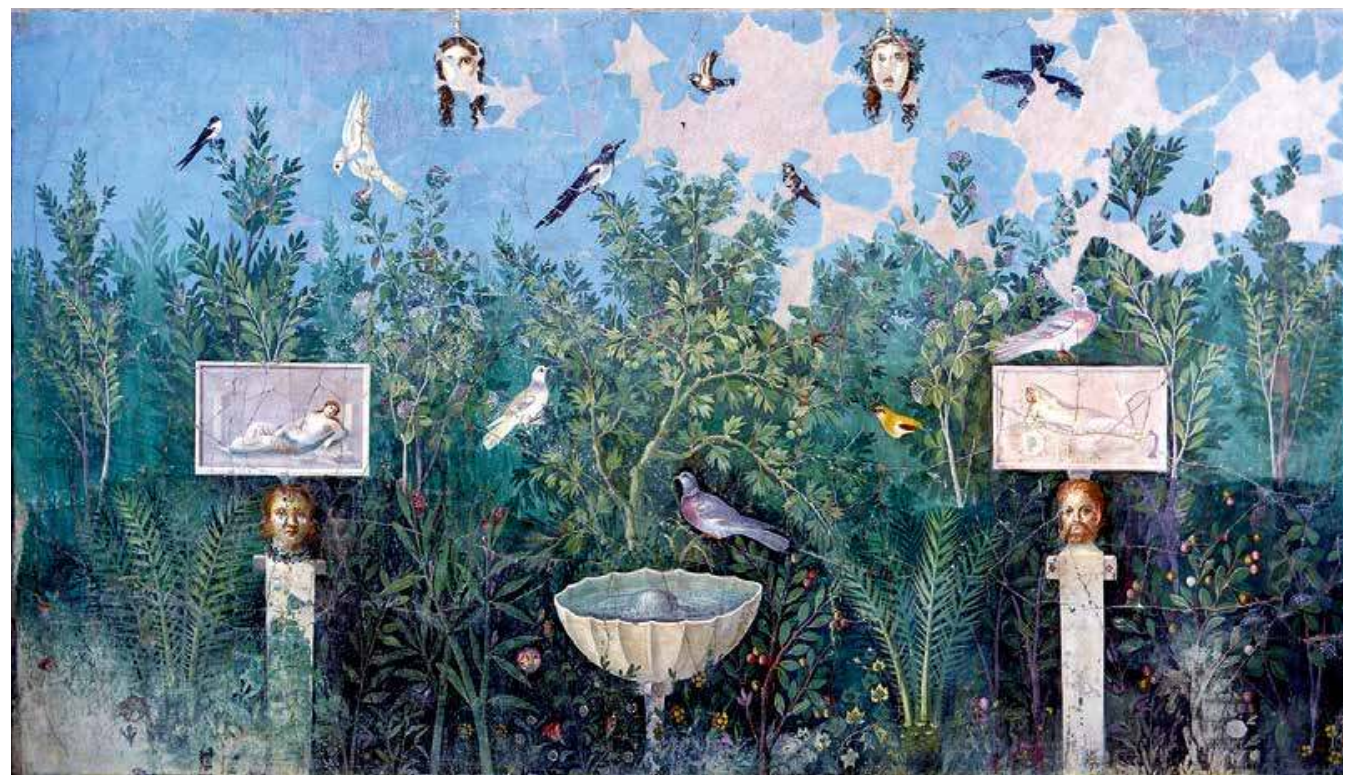

4. Détail du décor peint de la chambre 32, Pompéi, maison du Bracelet d'or, troisième style, Naples, Museo Archeologico Nazionale.

élégant aux affaires quotidiennes ", même si elle est sommaire, oblige à prendre en compte des niveaux différents, mais entremêlés, de réception et de perception.

La prédilection pour les surfaces planes et l'introduction d'une image dans une aedicula, petite structure à forme de pavillon placée au centre de la paroi, semble indiquer que le goût a évolué vers un schéma clair et intelligible à point focal unique. La structure des pavements en mosaïque évolue de manière similaire ${ }^{19}$. On a observé dans différentes maisons de Pompéi et des sites avoisinants la présence d'images quasi identiques, ce qui semble indiquer qu'un nombre limité de thèmes et d'iconographies à succès circulaient sous forme de livres de modèles et de dessins d'atelier ${ }^{20}$.

Ces répétitions et la variété du catalogue peuvent bien entendu être interprétées à différents niveaux. Quelle est la part du prescrit et du contrôle social dans le choix de l'imagerie destinée à la sphère privée ? Clarke estime qu'il faut se tourner vers le " grand art» officiel - les monuments publics, les portraits impériaux - pour voir la propagande augustéenne à l'œuvre, tandis que les décors des intérieurs privés semblent davantage refléter des tendances générales lancées par les architectes et décorateurs chargés d'embellir les demeures des élites et des classes moyennes romaines. Ces derniers connaissent sans aucun doute les modèles et les motifs introduits par les artistes de cour, et sont conscients de leur dimension idéologique. Malgré tout, l'assimilation de ces motifs par le grand public se déroule selon un rythme qui est fonction de la circulation des artistes et de la disponibilité des livres de modèles. De surcrôit, la diffusion du décor de troisième style autour de la baie de Naples ne s'est peut-être pas produite partout au même rythme. Les édifices s'adaptent au nouveau style et sont rénovés de manière différente et à des moments différents ; on conserve souvent les anciennes décorations des premier et deuxième styles, qui donnent une allure de vénérable ancienneté à la demeure et à la famille qui l'occupe ${ }^{21}$. En d'autres termes, la diffusion du décor de troisième style à Pompéi et dans les villes vésuviennes constitue les étapes ultimes dans l'histoire de cette longue "vague de mode ", celles de l'appropriation et de l'émulation. Les motifs rendus familiers par la propagande impériale ont désormais perdu tout lien direct avec les " grands " monuments ou avec les idées et les valeurs prônées par le prince. "Tout ce qui est arrivé sous le règne d'Auguste n'est pas nécessairement arrivé à cause d'Auguste", pour reprendre la formule de Karl Galinsky ${ }^{22}$.

\section{Images de la vie privée}

Les analyses de Clarke démontrent implicitement que la fonction et l'usage sont des facteurs déterminants lorsqu'il s'agit de choisir le sujet et le ton des décors privés. L'émergence du nouveau style dans la conception des peintures murales et des 
pavements de mosaïque s'accompagne d'une évolution architecturale notable, qui concerne notamment les espaces ouvrant sur un péristyle ou sur une terrasse donnant sur un paysage ${ }^{23}$. C'est au cours de cette période que les propriétaires de somptueuses demeures telles que la villa des Mystères se font construire des vérandas entourées de portiques et de petites pièces séparées, percées de larges ouvertures offrant une vue dégagée sur la mer. L'innovation que constituent les oeci cyziceni, dotés d'ouvertures sur les quatre côtés, et souvent flanqués de cubicula séparés, de taille plus petite, témoigne pareillement du goût pour l'isolement et le contact avec la nature ${ }^{24}$. S'il serait exagéré de dire que la période augustéenne a "inventé " la vie privée, Kristina Milnor a toutefois montré que c'est au cours de ces quelques décennies de l'histoire romaine que s'inventent de nouvelles stratégies visant à ménager une place à l'intimité et à la représenter.

Tandis que Wallace-Hadrill s'intéresse à l'ensemble des objets domestiques, et que Clarke se concentre sur le système de décoration du troisième style, Milnor se penche sur un aspect encore plus spécifique de l'habitat, à savoir les scènes mythologiques peintes et le choix des sujets. Certains exemples semblent témoigner d'une volonté délibérée d'affecter un "genre " aux intérieurs en y déployant des images de femmes vertueuses tirées des mythes grecs. La question centrale qui se pose ici est également au cœur des analyses de Wallace-Hadrill et de Clarke. L'imagerie visuelle est-elle essentiellement destinée à prêcher des leçons morales - de pudor et de pietas - aux hommes et aux femmes qui occupent ces espaces ? On note à cet égard que nombre de peintures mythologiques de Pompéi portent sur des formes transgressives du désir, plus que sur les valeurs et les idéaux de la famille romaine traditionnelle, s'inspirant davantage des Métamorphoses d'Ovide que du programme moral d'Auguste. Les qualités féminines et l'éloge de la « femme vertueuse » ne forment ainsi que l'un des discours qui coexistent dans la fabrique sociale de la demeure romaine. À en juger par la structure de l'habitat, où le public et le privé (ou plutôt le social et l'intime) se chevauchent davantage que ne l'indiquent les descriptions de Vitruve, il paraît difficile d'affirmer que les messages à portée sociale visent un groupe unique du foyer. Si la représentation et l'encadrement idéologique de la vie de famille constituent un instrument capital de la propagande et de l'image augustéennes, le fait que l'on observe des sujets similaires dans les maisons de la classe moyenne de Pompéi ne signifie en rien que ces canons officiels de moralité sont acceptés en bloc et sans réserve.

L'analyse de Milnor, qui se concentre sur les sources textuelles et juridiques concernant la vie privée des familles romaines, ouvre la voie à de nouvelles recherches sur le rôle de l'architecture privée dans la renovatio des valeurs traditionnelles de la famille romaine à l'époque d'Auguste. Cette architecture n'est-elle qu'un mode de représentation d'un nouveau système de relations ? Ou bien a-t-elle donné naissance à de nouveaux comportements en réorganisant l'expérience domestique ?

\section{Le miroir de la culture matérielle}

Toutes ces questions montrent que, trente ans après les analyses de Zanker sur l'imagerie littéraire et visuelle de la période augustéenne, il est encore difficile de déterminer avec précision les facteurs et les choix qui ont entraîné un bouleversement des goûts et des pratiques. De manière générale, la culture matérielle porte les traces des différentes interprétations appliquées au présent comme au passé et aux fonctions qu'on leur attribue. Mais rares sont les autres périodes de l'histoire où l'on observe pareille volonté délibérée d'assimiler les vestiges du passé dans la Dingwelt du présent. Dans un dense article publié en 2001, Annette Haug affirmait que l'ère d'Auguste offrait l'occasion idéale d'étudier la manière dont " le traitement des biens culturels [...] équivaut au traitement de l'histoire ". La renovatio (la reconquête du passé) en tant que concept politique distinct exige une reconstitution délibérée du passé, considéré à la fois comme différent du présent, et essentiel pour modeler celui-ci ${ }^{25}$. Les références non intentionnelles et conscientes - tradition et mémoire - se conjuguent pour donner expression à l'idéologie de la renovatio et au discours sur la culture matérielle qui en est le corollaire.

L'imagerie diffusée par Auguste et son cercle fait indéniablement un usage habile des artefacts afin de fournir divers points de références à l'interprétation du passé comme du présent. Le Cupidon ailé chevauchant un dauphin placé au pied de l'Auguste de Prima Porta, statue plus grande que nature de l'empereur, pourrait faire référence à la fois au passé récent, de mémoire vivante, et au lointain passé mythologique, accessible seulement par le biais de la mémoire collective (musées du Vatican, Braccio 
nuovo, inv. $\mathrm{n}^{\circ} 2290$ ). Ainsi, les strates du passé se combinent pour construire une représentation du présent. Attribut du dieu Neptune, le dauphin fait allusion aux victoires navales d'Auguste, et surtout à la fameuse bataille d'Actium, qui a permis d'annexer l'Égypte et de pacifier l'Empire. Mais le public romain aurait aussi vu dans le Cupidon une revendication généalogique, puisque la gens Iulia est censée descendre de Vénus ${ }^{26}$. Le dauphin renforce ce lien en faisant allusion à la déesse elle-même, née de la mer (fig. 5).

Comment tout ceci permet-il de mieux comprendre les significations et les implications idéologiques - le cas échéant - d'images et de contextes très éloignés du patronage impérial ou qui, s'ils ont un lien avec les plus hauts rangs de la société romaine (comme par exemple à la villa de Boscotrecase), n'étaient pas utilisés lors de cérémonies officielles? Les données examinées par Wallace-Hadrill, Clarke et Milnor sous des angles différents semblent indiquer que le goût et la mode suivent des rythmes parfois différents de ceux de la propagande impériale et qui, autrement dit, ne correspondent pas à un schéma d'imposition de modèles par le pouvoir. Toutefois, afin de justifier pleinement les évolutions qui se produisent en matière de conception et de style, spectaculaires et largement répandues (mais sans doute moins rapides qu'on ne le croit généralement), il faudrait, au-delà des seules considérations formelles du décorateur et de l'architecte, proposer une explication qui envisage une forme de dialogue avec les formes impériales et les sources de savoir prédominantes.

\section{Ce texte a été traduit par Françoise Jaouën.}

Anna Anguissola, Università di Pisa, Dipartimento di Civiltà e Forme del Sapere anna.anguissola@unipi.it

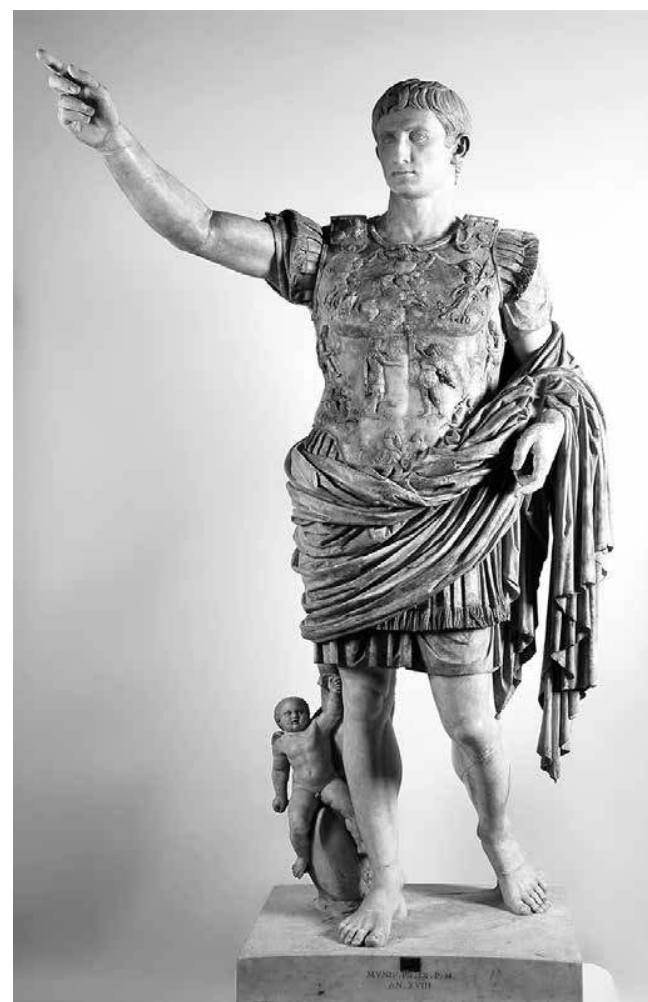

5. Statue cuirassée de l'Auguste de Prima Porta, fin du I $^{\text {er }}$ siècle av. notre ère, Cité du Vatican, Musei Vaticani, Braccio Nuovo.
1. "[O]ptimism and confidence which, as it was given expression by Augustan poets, left for posterity the concept of the Saeculum Augustum ", Paul Zanker, The Power of Images in the Age of Augustus, Alan Shapiro (trad. angl.), Ann Arbor, 1988, p. 1 [éd. orig. : Augustus und die Macht der Bilder, Munich, 1987].

2. Sur l'idéologie augustéenne de la renovatio et son impact sur la création littéraire et artistique, voir Frédéric Hurlet, Bernard Mineo (dir.), Le principat d'Auguste: réalités et représentations du pouvoir autour de la «Res publica restituta ", Rennes, 2009

3. Tonio Hölscher, The Language of Images in Roman Art, Anthony Snodgrass, Annemarie Künzl-Snodgrass [trad. angl.], Cambridge, 2004 [éd. orig. : Römische Bildsprache als semantisches System, Heidelberg, 1987].

4. Le programme architectural d'Auguste à Rome a fait l'objet de nombreuses études ; voir notamment Diane Favro, The Urban Image of Augustan Rome, Cambridge/ New York, 1996 ; Kathleen S. Lamp, A City of Marble. The Rhetoric of Augustan Rome, Columbia, Caroline du Sud, 2013 ; Lothar Haselberger, Urbem adornare. Die Stadt Rom und ihre Gestaltumwandlung unter Augustus/Rome's Urban Metamorphosis under Augustus, Portsmouth, Rhode Island, 2007.

5. Antony J. S. Spawforth, Greece and the Augustan Cultural Revolution, Cambridge, 2012. Sur les liens entre Auguste et les provinces, voir également Detlev Kreikenbom, 
Karl-Uwe Mahler, Patrick Schollmeyer, Thomas M. Weber (dir.), Augustus - Der Blick von Außen. Die Wahrnehmung des Kaisers in den Provinzen des Reichs und Nachbarstaaten, Wiesbaden, 2008.

6. Les œuvres d'art et les édifices peuvent faire l'objet d'une commande directe, mais Zanker met en avant l'élément de spontanéité : "nothing was explecitly prescribed, nothing supervised, and there were no advertising campaigns [rien n'était explicitement prescrit, rien n'était encadré, et il n'y avait aucune campagne de promotion] " (Zanker, [1987] 1988, cité n. 1, p. 338).

7. Sur les décors de troisième style, voir Frédéric L. Bastet, Mariette de Vos, Proposta per una classificazione del terzo stile pompeiano, Rome, 1979.

8. Sur la villa de Boscotrecase, l'ouvrage de référence est celui de Peter H. von Blanckenhagen, Christine Alexander, The Augustan Villa at Boscotrecase, Mayence, 1990.

9. Dans un célèbre compte rendu, Wallace-Hadrill qualifiait de " Rome's cultural revolution [révolution culturelle romaine] " les changements culturels et sociaux introduits sous le règne d'Auguste ("Rome's Cultural Revolution ", dans The Journal of Roman Studies, $\mathrm{n}^{\circ}$ 79, 1989, p. 157-164. Il estimait que le livre de Zanker ([1987] 1988, cité n. 1) était l'ouvrage le plus important sur la Rome augustéenne publié depuis The Roman Revolution de Sir Ronald Syme (1939). Il est impossible d'évoquer ici en détail la portée des analyses de Wallace-Hadrill concernant d'autres aspects de la culture augustéenne; voir le compte rendu de Robin Osborne, Caroline Vout, "A Revolution in Roman History ", dans Journal of Roman Studies, $n^{\circ}$ 100, 2010 , p. 233-245.

10. Sur les triomphes romains et le pillage des œuvres grecques, voir Margaret Miles, Art as Plunder: The Ancient Origins of Debate about Cultural Property, Cambridge/ New York, 2008.

11. Les complexes ramifications historiques et philosophiques des concepts de goût et de mode ne peuvent être citées ici ; Jukka Gronow fournit un large aperçu des textes consacrés au sujet dans The Sociology of Taste, Londres/New York, 1997.

12. Salvatore Settis fournit une excellente introduction au patronage impérial et aux peintures de jardins à Rome dans Le pareti ingannevoli: la Villa di Livia e la pittura di giardino, Milan, 2002. Annamaria Ciarallo analyse les liens entre l'art du topiaire et la rhétorique du luxe dans "Espressioni del lusso dal mondo naturale ", dans Elena Fontanella (dir.), Luxus. Il piacere della vita nella Roma imperiale, Rome, 2009. Sur le goût pour les grottes naturelles et artificielles, voir également Henri Lavagne, "Operosa antra." Recherches sur la grotte à Rome de Sylla à Hadrien, Rome, 1989 (particulièrement p. 439-511).

13. Voir Rosaria Ciardiello, «VI 17 Insula Occidentalis 42. Casa del Bracciale d'Oro ", dans Masanori Aoyagi et Umberto Pappalardo (dir.), Pompei (Regiones VI-VII). Insula Occidentalis, Naples, 2006, p. 69-256.

14. Voir Eric M. Moormann, "Giardini ed altre pitture nella Casa del futteto e nella Casa del Braciale d'oro a Pompei ", dans Mededelingen van het Nederlands Instituut te Rome, $\mathrm{n}^{\circ}$ 54, 1995, p. 214-227 ; Anna Anguissola, Intimità a Pompei. Riservatezza, condivisione e prestigio negli ambienti ad alcova di Pompei, Berlin/New York, 2010, p. 323-326.

15. Sur l'introduction et la vogue de l'imagerie égyptienne, voir Miguel John Versluys, Aegyptiaca Romana. Nilotic Scenes and the Roman Views of Egypt, Leyde, 2002 ; Laurent Bricault, Miguel John Versluys, Paul G. P. Meyboom (dir.), Nile into Tiber. Egypt in the Roman World, Leyde/ Boston, 2007 (en particulier p. 113-208), et Molly Swetnam-Burland, Egypt in Italy. Visions of Egypt in Roman Imperial Culture, Cambridge, 2015.

16. Sur le retour en arrière vers le classicisme dans l'art romain, voir Jas Elsner, "Classicism in Roman Art ", dans James I. Porter (dir.), Classical Pasts: The Classical Traditions of Greece and Rome, Princeton, 2005, p. 270-297.

17. Sur le concept de luxus, voir Karl Wilhelm Weeber, Luxus im alten Rom, Darmstadt, 2003. Voir également Stefano Tortorella, "Il lusso nella vita privata come espressione di una aristocrazia al potere ", dans Eugenio La Rocca, Claudio Parisi Presicce, Annalisa Lo Monaco, Cécile Giroire, Daniel Roger (dir.), Augusto, Milan, 2013, p. 252-269.

18. " [Are they] preaching an Augustan ideology of neoclassicism, piety, and restraint, or do they simply express a new, elegant, and much copied fashion interior design? " CLARKE, 2007, p. 265.

19. Voir Anguissola, 2010, citée n. 14, p. 302-303, et 487 488 , n. 513.

20. Voir notamment von Blanckenhagen, Alexander, 1990, cités n. 8, p. 42-43 et 57-58.

21. John R. Clarke, "The Early Third Style at the Villa of Oplontis ", dans Mitteilungen des Deutschen Archäologischen Instituts, Römische Abteilung, $\mathrm{n}^{\circ}$ 94, 1987, p. 267-294.

22. Karl Galinsky, "Introduction ", dans The Cambridge Companion to the Age of Augustus, Cambridge/New York, 2007, p. 1-9 (en particulier p. 6).

23. Sur la dimension privée des peintures romaines à la fin de la période républicaine et au début de l'Empire, voir également Gilles Sauron, Dans l'intimité des maîtres $d u$ monde. Les décors privés des Romains, Paris, 2009.

24. Voir Anguissola, 2010, citée n. 14, p. 218-229.

25. Annette Haug, "Constituting the Past, Forming the Present. The Role of Material Culture in the Augustan Period ", dans Journal of the History of Collections, 13 , $\mathrm{n}^{\circ} 2,2001, \mathrm{p} .111$. Sur les liens entre identité et culture matérielle à la fin de la période républicaine, voir Miguel John Versluys, "Material Culture and Identity in the Late Roman Republic (200 BC-20 BC) ", dans Jane DeRose Evans (dir.), Blackwell Companion to the archaeology of the Roman Republic, Malden, Mass./Oxford/Chichester, 2013, p. 429-440.

26. Sur les ramifications idéologiques et politiques de la généalogie d'Auguste, voir Zanker, (1987) 1988, cité n. 1, p. 193-210, 215-227. Sur l'Auguste de Prima Porta et le système de références mis en œuvre par les attributs, le type corporel, les thèmes, et les reliefs du plastron, voir Michael Squire, "Embodied Ambiguities on the Prima Porta Augustus ", dans Art History, n 36, 2013, p. 242-279. 